\title{
ArcheoSciences
}

Revue d'archéométrie

\section{Découverte exceptionnelle de restes de Crevettes (Crustacés Décapodes) dans les niveaux du port romain de Ratiatum (Rezé, Loire-Atlantique)}

Exceptional discovery of marine shrimp remains (Crustacea decapoda) in the levels of the Roman harbour area of Ratiatum (Rezé, Loire Atlantique)

\section{Aurélia Borvon et Yves Gruet}

\section{OpenEdition \\ Journals}

Édition électronique

URL : https://journals.openedition.org/archeosciences/5062

DOI : 10.4000/archeosciences.5062

ISSN : 2104-3728

\section{Éditeur}

Presses universitaires de Rennes

Édition imprimée

Date de publication : 27 juin 2018

Pagination : 7-15

ISBN : 978-2-7535-7587-5

ISSN : $1960-1360$

\section{Référence électronique}

Aurélia Borvon et Yves Gruet, « Découverte exceptionnelle de restes de Crevettes (Crustacés

Décapodes) dans les niveaux du port romain de Ratiatum (Rezé, Loire-Atlantique) », ArcheoSciences [En ligne], 42-1 | 2018, mis en ligne le 27 juin 2020, consulté le 27 janvier 2022. URL : http://

journals.openedition.org/archeosciences/5062; DOI : https://doi.org/10.4000/archeosciences.5062 


\title{
Découverte exceptionnelle de restes de Crevettes (Crustacés Décapodes) dans les niveaux du port romain de Ratiatum (Rezé, Loire-Atlantique)
}

\author{
Exceptional Discovery of Marine Shrimp Remains (Crustacea decapoda) \\ in the Levels of the Roman Harbour Area of Ratiatum (Rezé, Loire Atlantique)
}

\author{
Aurélia Borvon ${ }^{a}$ et Yves Gruet ${ }^{b}$
}

\begin{abstract}
Résumé : Des restes de crevettes (Crustacés Décapodes) ont été découverts à l'occasion de fouilles archéologiques programmées réalisées au sein du quartier portuaire romain de Ratiatum (Rezé, Loire-Atlantique). Issus de deux lots datés de la première moitié du II $^{\mathrm{e}}$ siècle et de la première moitié du $\mathrm{III}^{\mathrm{e}}$ siècle apr. J.-C., les carapaces de ces petits animaux sont indétectables sans prélèvement et tamisage fin des sédiments (maille $1,1 \mathrm{~mm},-20$ litres). La présence de ces fragments de crevettes est exceptionnelle en contexte archéologique : très fragiles, leur préservation est liée à l'excellente conservation des vestiges en milieu humide. Les restes de deux espèces ont pu être identifiés, grâce à la morphologie de leur rostre (prolongement rigide de la tête) pour la crevette blanche Palaemon longirostris, et de leur telson (partie terminale postérieure du corps des Arthropodes) pour la crevette grise Crangon crangon. Les éléments des premières sont fréquents, avec 85 rostres dénombrés, tandis que les secondes sont identifiées seulement par trois morceaux de telson. Des relations biométriques établies à partir de référentiels actuels permettent de reconstituer la taille (longueur) des individus de Rezé, à partir des mensurations des pièces de l'exosquelette mentionnées. L'association systématique avec de petits poissons très probablement utilisés pour la confection de sauce de poissons atteste de la probable participation des crevettes à ce type de préparation. L'écologie de la principale espèce identifiée $P$. longirostris témoigne d'une activité de pêche réalisée dans une zone estuarienne d'un fleuve comme la Loire.
\end{abstract}

\begin{abstract}
Shrimp remains (Crustacea decapoda) have been discovered in programmed archaeological excavations in the Roman harbour area of Ratiatum (Rezé, Loire-Atlantique). Coming from two sets of shrimp remains dating from the first half of the $2^{\text {nd }}$ century and from the first half of the $3^{\text {rd }}$ century A.D., the parts of carapaces from these small animals can only be detected after carefully sieving about 20 litres of sediment with a $1,1 \mathrm{~mm}$ square mesh. The discovery of these shrimp fragments is exceptional at an archaeological site and their presence is linked to a good preservation in a damp environment. The morphology of the rostrum helps us to determine Palaemon longirostris with four spines under the rostrum whilst the morphology of the telson (the part at the rear end of the body of arthropods) makes the sand shrimp (Crangon crangon) clearly identifiable. The number of Palaemon longirostris rostrums (85) is quite considerable, nevertheless there are only three Crangon telsons. Biometrical relations (total lengths related to distance between tooth 1 and 4 under the rostrum) established from current-day shrimps help us to reconstitute the original lengths of the crustacea of Rezé by measuring the parts of the exoskeleton mentioned. The fact that pieces of shrimps are systematically associated with small fish used in preparing fish sauces suggests that these shrimps were probably used in this type of preparation too. The ecology of the main species Palaemon longirostris shows that they were caught in an estuary such as that of the Loire.
\end{abstract}

Mots clés : Crevettes, Crustacés Décapodes, Antiquité, Rezé Saint-Lupien, Loire-Atlantique, biométrie, pêche.

Keywords: shrimps, Crustacea decapoda, Antiquity, Rezé Saint-Lupien, Loire-Atlantique, biometry, fishing.

\footnotetext{
a UMR 7041 ArScAn, Équipe Archéologies Environmentales - Laboratoire d'Anatomie Comparée, ONIRIS (École vétérinaire, Agroalimentaire et de l'Alimentation, Nantes, Loire-Atlantique) - GEROM Groupe d'Études Remodelage Osseux et Biominéraux - Angers. (aureliaborvon@gmail.com)

${ }^{b}$ Maître de Conférences, retraité de la Faculté des Sciences de l'université de Nantes. (achil.lemeur@wanadoo.fr)
} 


\section{INTRODUCTION}

Entre 2005 et 2016, le port antique de Ratiatum (Rezé, quartier Saint-Lupien, Loire-Atlantique), situé en rive sud de la Loire (figure 1), a fait l'objet de fouilles archéologiques programmées. Elles portaient sur les aménagements de berges et leur environnement. Ce secteur s'urbanise à partir des années 20-50 de notre ère et l'occupation se poursuit jusqu'au milieu du $\mathrm{III}^{\mathrm{e}}$ siècle (Guitton et Mouchard, 2016). Presque tous les secteurs de fouille ont livré des restes de Vertébrés (Borvon, 2016). En revanche, les restes d'Invertébrés Arthropodes, tels les fragments de Crevettes (Crustacés Décapodes), sont bien plus rares. Très fragiles, la présence de ces vestiges est exceptionnelle en contexte archéologique. Leur préservation est liée à la très bonne conservation d'une partie des vestiges en milieu humide. L'objectif est ici de déterminer ces restes de crevettes, de reconstituer la taille (longueur) des animaux, ainsi que d'émettre des hypothèses sur les raisons de leur présence dans cet environnement portuaire antique et sur les lieux de pêche potentiels.

\section{Matériel et MÉThodes}

Les restes de crevettes proviennent des secteurs 14 et 18 de la zone 4, tous deux localisés en pied de pente au contact des aménagements portuaires antiques. Les vestiges sont issus de deux prélèvements de sédiments réalisés en milieu humide lors de la campagne de fouilles de 2014 au sein des Unités stratigraphiques (Us) 40760 et 43357 . L'Us 40760 correspond à des rejets liés à la dernière phase d'occupation de l'un des caissons portuaires (CAI 12) et mêlés à un apport fluviatile de sable de Loire grossier à fin, peu compact et homogène. Ce niveau est daté de la première moitié $\mathrm{du} \mathrm{II}^{\mathrm{e}}$ siècle apr. J.-C. (Guitton et Mouchard, 2015). L'Us 43357 est quant à elle une couche d'argile grise très plastique et compacte comprenant de très nombreux débris de végétaux et de galets de rivières. Elle serait datée de la première moitié du III $^{\mathrm{e}}$ siècle apr. J.-C. Le volume prélevé est respectivement de 10 litres et 8,4 litres. Les sédiments ont été lavés sur des tamis de mailles de $4 \mathrm{~mm}$ et 1,1 $\mathrm{mm}$ (Borvon, 2016).

\section{Identification des crevettes actuelles de la région}

Des crevettes actuelles ont été prélevées de manière à fournir des référentiels auxquels se reporter pour les comparer

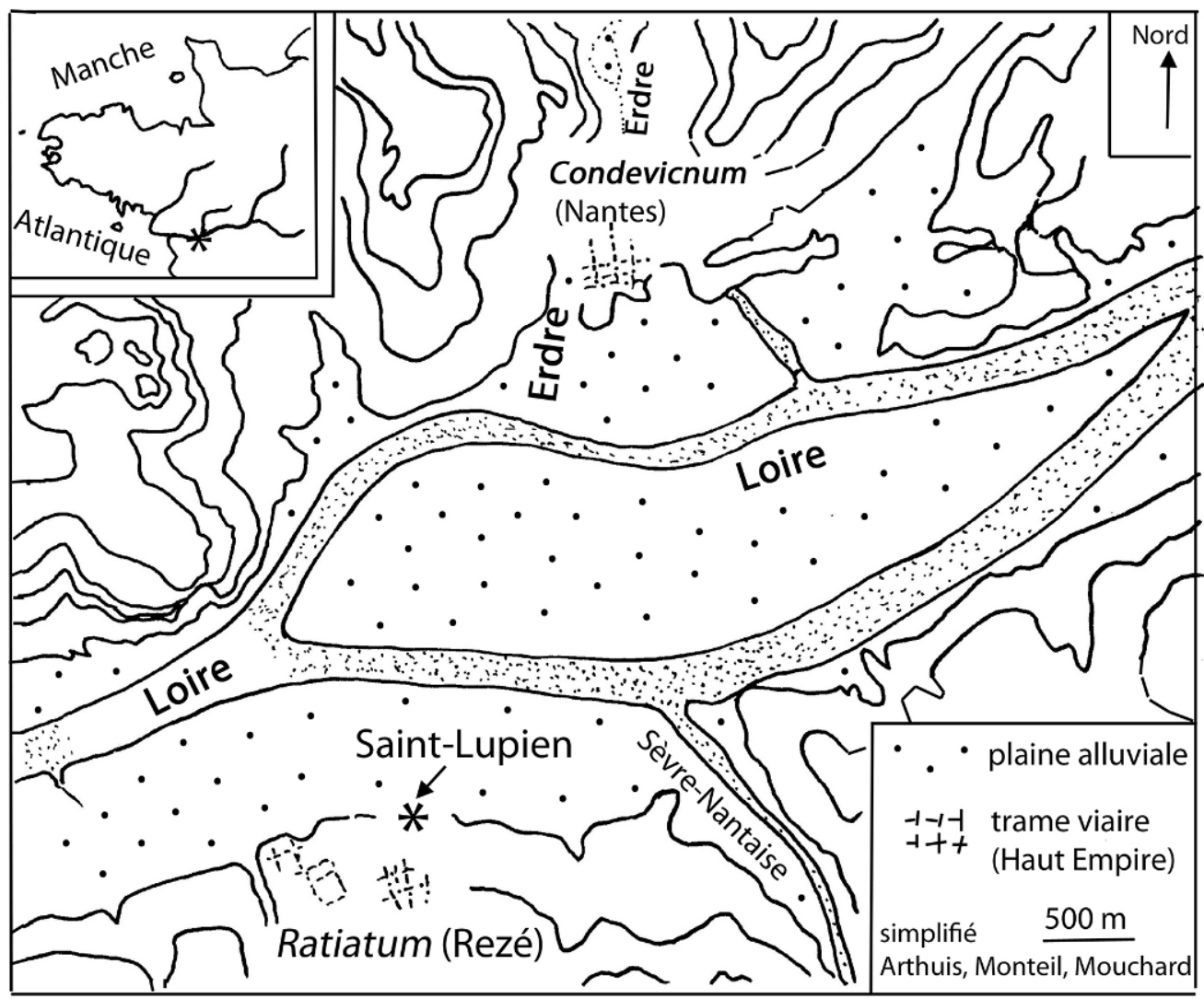

Figure 1 : Localisation de l'agglomération antique de Rezé (Loire-Atlantique) dans l'estuaire de la Loire (d'après R. Arthuis, M. Monteil et J. Mouchard, modifié).

Figure 1: Location of the antique town of Rezé (LoireAtlantique) in the Loire estuary (from R. Arthuis, M. Monteil and J. Mouchard, modified). 
avec les restes archéologiques. Ces crustacés sont identifiés grâce à des ouvrages de faunistique comme G. Smaldon (1979), R. Zariquiey Alvarez (1968) ou E.L. Bouvier (1940). L'identification des espèces utilise différents caractères morphologiques, dont ceux du rostre (tableau 1).

\section{Quelques données sur la bio-écologie des différentes espèces de crevettes de la région}

Dans le tableau 1, les caractéristiques du milieu de vie des principales espèces de la région sont présentées. La crevette grise Crangon crangon vit sur toutes les étendues sableuses des estrans et en zone subtidale. Elle remonte bien aussi en estuaire interne de la Loire jusqu’à Paimboeuf environ. Les Palaemon longirostris se rencontrent en revanche uniquement dans les estuaires (Aurousseau, 1984; Béguer, 2009). À proximité de Nantes, elles vivent dans l'estuaire interne de la Loire entre Paimbœuf et Mindin et à l'amont de Paimbœuf (Marchand, 1981; Marchand et Alliot, 1981). Elles sont en revanche absentes des marais briérons où une autre crevette d'eau saumâtre Palaemonetes varians vit en grand nombre (Gruet et Baudet, 2007).

\section{Morphologie de la carapace des crevettes et détermination des restes de Rezé}

Les crevettes, comme les autres crustacés, sont caractérisées par un exosquelette ou carapace. De l'avant vers l'arrière, cette carapace comprend un rostre terminant le céphalothorax, puis un abdomen avec un telson à son extrémité (figure 2). Généralement, c'est la morphologie du rostre qui est utile pour déterminer les échantillons archéologiques (tableau 1, figure 3). Cependant, certaines espèces comme les crevettes grises présentent un rostre très court. Elles possèdent en revanche un telson caractéristique.

\section{Mesures des crevettes actuelles et des restes de Rezé, reconstitution des longueurs des individus archéologiques}

Les rostres et les telsons de Ratiatum ont été dessinés à la chambre claire. Les mesures réalisées sur ces dessins sont converties en valeurs réelles en fonction du grossissement adopté. Il en a été de même pour les rostres d'une série de référence de Palaemon longirostris actuelles, ainsi que pour les telsons de Crangon crangon.

\begin{tabular}{|c|c|c|c|c|c|c|c|c|c|}
\hline & $\begin{array}{l}\text { Palaemon } \\
\text { adspersus }\end{array}$ & $\begin{array}{l}\text { Palaemon } \\
\text { elegans }\end{array}$ & $\begin{array}{l}\text { Palaemon } \\
\text { longirostris }\end{array}$ & $\begin{array}{c}\text { Palaemon } \\
\text { serratus }\end{array}$ & $\begin{array}{c}\text { Palaemonetes } \\
\text { varians }\end{array}$ & $\begin{array}{l}\text { Crangon } \\
\text { crangon }\end{array}$ & $\begin{array}{l}\text { Pandalina } \\
\text { brevirostris }\end{array}$ & Processa edulis & $\begin{array}{l}\text { Atyaephyra } \\
\text { desmarestii }\end{array}$ \\
\hline & - & $\begin{array}{l}\text { petite crevette } \\
\text { rose }\end{array}$ & $\begin{array}{l}\text { crevette } \\
\text { blanche }\end{array}$ & $\begin{array}{l}\text { crevette rose, } \\
\text { bouquet }\end{array}$ & $\begin{array}{l}\text { crevette de } \\
\text { marais }\end{array}$ & $\begin{array}{l}\text { crevette grise, } \\
\text { de sable }\end{array}$ & - & - & $\begin{array}{l}\text { Caridine, } \\
\text { crevette d'eau } \\
\text { douce }\end{array}$ \\
\hline ROSTRE & $\begin{array}{l}\text { droit } \\
3 \text { dents ven- } \\
\text { trales } \\
(2-4)\end{array}$ & $\begin{array}{l}\text { droit } \\
\text { peu recourbé } \\
\text { vers le haut } \\
3 \text { dents ven- } \\
\text { trales } \\
(2-4)\end{array}$ & $\begin{array}{l}\text { droit } \\
\text { peu recourbé } \\
\text { vers le haut } \\
3 \text { ou } 4 \text { dents } \\
\text { ventrales } \\
(3-5)\end{array}$ & $\begin{array}{l}\text { nettement } \\
\text { recourbé vers } \\
\text { le haut } \\
4 \text { ou } 5 \text { dents } \\
\text { ventrales }\end{array}$ & $\begin{array}{l}\text { droit } \\
2 \text { dents ven- } \\
\text { trales } \\
(0-1-3-4)\end{array}$ & $\begin{array}{l}\text { rostre très } \\
\text { court }\end{array}$ & $\begin{array}{l}\text { droit } \\
2 \text { ou } 3 \text { dents } \\
\text { ventrales }\end{array}$ & $\begin{array}{l}\text { droit } \\
\text { COURT } \\
\text { vers le bas } \\
0 \text { dent ven- } \\
\text { trale }\end{array}$ & $\begin{array}{l}\text { droit } \\
\text { LONG } \\
\text { très nom- } \\
\text { breuses dents } \\
\text { dorsales }\end{array}$ \\
\hline $\begin{array}{l}\text { extrémité } \\
\text { du rostre }\end{array}$ & souvent bifide & souvent bifide & $\begin{array}{l}\text { occasionnelle- } \\
\text { ment bifide }\end{array}$ & $\begin{array}{l}\text { habituelle- } \\
\text { ment bifide }\end{array}$ & $\begin{array}{l}\text { occasionnelle- } \\
\text { ment bifide }\end{array}$ & & & & \\
\hline $\begin{array}{l}\text { salinité de } \\
\text { l'eau }\end{array}$ & eau marine & $\begin{array}{l}\text { supporte } \\
\text { un peu de } \\
\text { dessalure }\end{array}$ & $\begin{array}{l}\text { supporte bien } \\
\text { la dessalure }\end{array}$ & eau marine & $\begin{array}{l}\text { eau saumâtre : } \\
\text { de sursalée à } \\
\text { doux }\end{array}$ & eau marine & eau marine & eau marine & eau douce \\
\hline biotope & $\begin{array}{l}\text { milieu } \\
\text { rocheux } \\
\text { intertidal à } \\
\text { peu profond }\end{array}$ & $\begin{array}{l}\text { estrans } \\
\text { (mares) }\end{array}$ & $\begin{array}{l}\text { estuaires } \\
\text { peu profond }\end{array}$ & $\begin{array}{l}\text { milieu } \\
\text { rocheux : bas } \\
\text { de l'estran et } \\
\text { zone subtidale }\end{array}$ & $\begin{array}{l}\text { étiers et } \\
\text { marais sau- } \\
\text { mâtres } \\
\text { peu profond }\end{array}$ & $\begin{array}{l}\text { estran et } \\
\text { subtidal } \\
\text { sable ou vase }\end{array}$ & $\begin{array}{l}\text { estran et } \\
\text { subtidal } \\
\text { sable }\end{array}$ & $\begin{array}{l}\text { estran et } \\
\text { subtidal }\end{array}$ & $\begin{array}{l}\text { rivières lentes, } \\
\text { canaux } \\
\text { (végétation } \\
\text { aquatique) }\end{array}$ \\
\hline abondance & peu fréquente & très abondante & $\begin{array}{l}\text { abondante par } \\
\text { endroits }\end{array}$ & abondante & très abondante & abondante & peu fréquente & peu fréquente & $\begin{array}{l}\text { abondante par } \\
\text { endroits }\end{array}$ \\
\hline $\begin{array}{l}\text { distribu- } \\
\text { tion }\end{array}$ & $\begin{array}{l}\text { espèce tem- } \\
\text { pérée }\end{array}$ & $\begin{array}{l}\text { espèce tem- } \\
\text { pérée }\end{array}$ & $\begin{array}{l}\text { espèce tem- } \\
\text { pérée }\end{array}$ & $\begin{array}{l}\text { espèce tem- } \\
\text { pérée }\end{array}$ & $\begin{array}{l}\text { espèce tem- } \\
\text { pérée }\end{array}$ & $\begin{array}{l}\text { espèce tempé- } \\
\text { rée froide }\end{array}$ & & & $\begin{array}{l}\text { espèce intro- } \\
\text { duite }\end{array}$ \\
\hline
\end{tabular}

Tableau 1 : Caractères des rostres de quelques espèces littorales de crevettes du Golfe de Gascogne (Smaldon, 1979; Zariquiey Alvarez, 1968; Bouvier, 1940) et présentation des caractéristiques écologiques de ces espèces (Udekem d'Acoz, 1999; Noël, 1992).

Table 1: Characteristics of rostrums of some littoral shrimps from the Gulf of Biscaye (Smaldon, 1979; Zariquiey Alvarez, 1968; Bouvier, 1940), and ecological characters of these species (Udekem d'Acoz, 1999; Noël, 1992). 
longueur totale

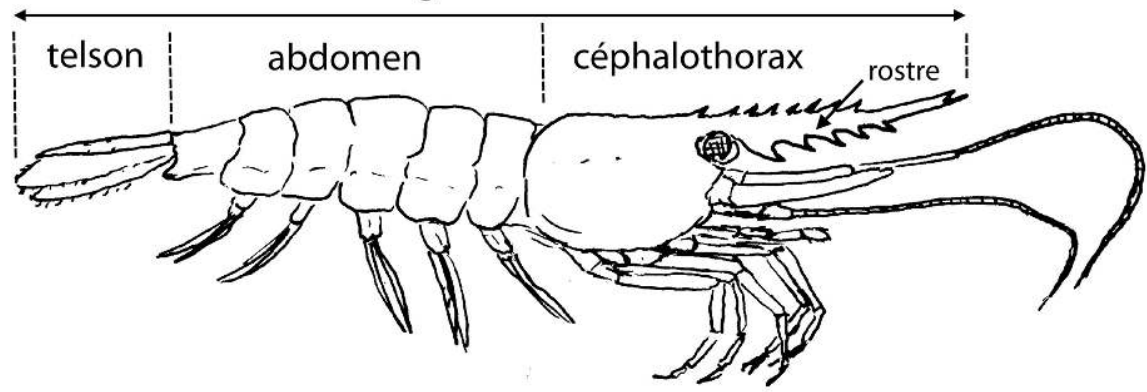

Figure 2 : Schéma d'une crevette vue de profil (dessin Y. Gruet).

Figure 2: Diagram of a shrimp profile view (drawing Y. Gruet).

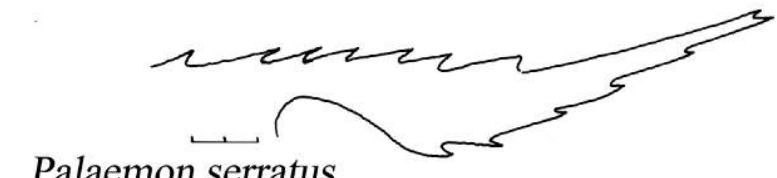

Palaemon serratus

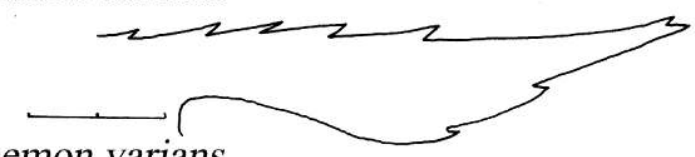

Palaemon varians
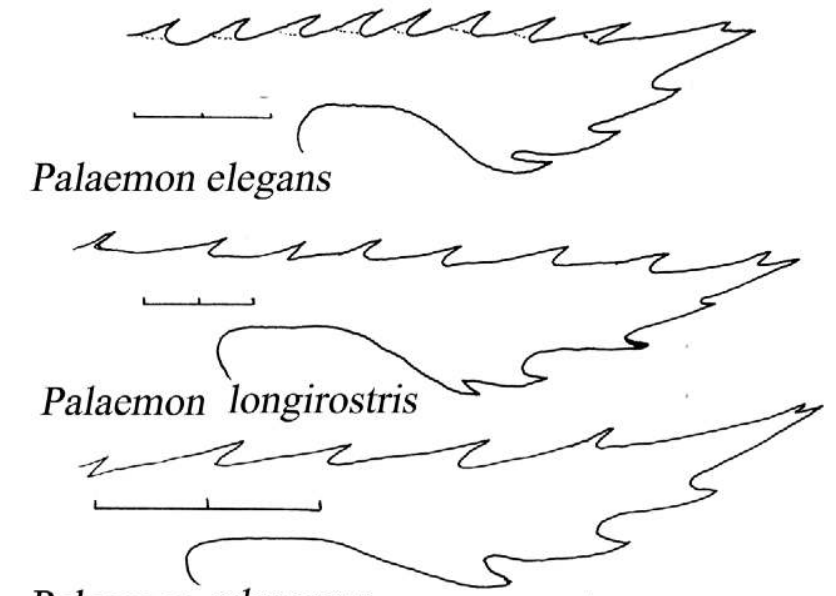

Palaemon adspersus

Figure 3 : Rostres de cinq espèces de crevettes actuelles et communes (dessin Y. Gruet). L'échelle est de $2 \mathrm{~mm}$.

Figure 3: Rostrums of five actual and common prawns (drawings Y. Gruet). Scale is $2 \mathrm{~mm}$.

De manière à déterminer la longueur des spécimens archéologiques, des relations biométriques pour des crevettes actuelles ont été établies. Les crustacés croissent de manière discontinue, car ils grandissent par mues successives tout au long de leur vie, avec un ralentissement à la fin. Les rostres et les telsons de crevettes actuelles sont mesurés ainsi que la longueur totale des individus. Ces caractères quantitatifs continus se déduisent l'un de l'autre par une liaison sta- tistique. Les ajustements linéaires sont examinés en calculant les coefficients de corrélation, qui seront d'autant plus valables qu'ils seront proches de 1 ou -1 (Schwartz, 1963).

La relation qui lie la distance entre les épines du rostre chez Palaemon longirostris et la longueur totale de la crevette, est linéaire et de type $\mathrm{y}=\mathrm{ax}+\mathrm{b}$. Il en est de même pour la relation entre la longueur du telson et la longueur totale de la crevette grise.

\section{RÉsultats}

\section{Identification des restes de crevettes de Rezé}

Les restes de crustacés sont variés, mais les rostres et les telsons sont les plus faciles à reconnaître et à identifier. Ce sont aussi ces pièces qui vont être utilisées pour la restitution des longueurs. D'après la forme générale (figures 3, 4) et le nombre de dents sous le rostre qui est de 4 (tableau 1), la plupart des crevettes de Rezé appartiennent à l'espèce Palaemon longirostris. Les telsons (figure 5) correspondent à ceux de crevettes grises Crangon crangon. Au total, sur 150 rostres, 85 appartiennent avec certitude à Palaemon longirostris. La fragmentation des autres pièces empêche d'être certain de leur appartenance spécifique (très probablement la même espèce). Trois telsons de Crangon crangon ont également été reconnus.

\section{Reconstitution des longueurs des crevettes de Rezé}

Les 48 rostres les mieux conservés de Palaemon longirostris, 38 dans l'Us 40760 et 10 dans l'Us 43357 permettent de mesurer les distances (d) séparant les dents 1 à 4 du dessous du rostre (figure 4). Ces mêmes longueurs ont été mesurées chez 54 individus actuels de P. longirostris dont la longueur totale $(\mathrm{Lt})$ est connue. La relation linéaire obtenue pour ces individus de référence est : 


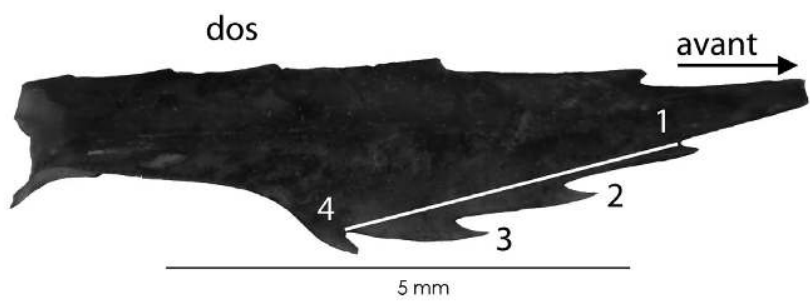

Figure 4 : Rostre d'une Crevette P. longirostris du site de Rezé (cliché A. Borvon). L'échelle fait $2 \mathrm{~mm}$.

Figure 4: Rostrum of a P. longirostris shrimp of the Rezé archaeological site (photo A. Borvon). Scale is $2 \mathrm{~mm}$.

Lt $($ en $\mathrm{mm})=\mathrm{d}(1$ à 4$) \times 7,80145-11,2654$, avec un coefficient de corrélation $\mathrm{r}=0,93$ (figure 6).

Cette relation permet de calculer la longueur totale des crevettes de Rezé (figure 7). Les longueurs reconstituées correspondent à des tailles comprises entre 25 et $45 \mathrm{~mm}$ de long pour $94 \%$ des 48 individus.

Pour Crangon, la relation biométrique entre la longueur totale (Lt) de la crevette et la longueur de son telson (Ls) est la suivante :

$\mathrm{L}=5,177 \times \mathrm{Ls}+3,52514$; avec $\mathrm{r}=0,98$ (figure 8).

Les trois telsons archéologiques (Us 40760) appartenaient à des crevettes de $32,7 \mathrm{~mm}, 41,5 \mathrm{~mm}$ et $46,6 \mathrm{~mm}$ de long.

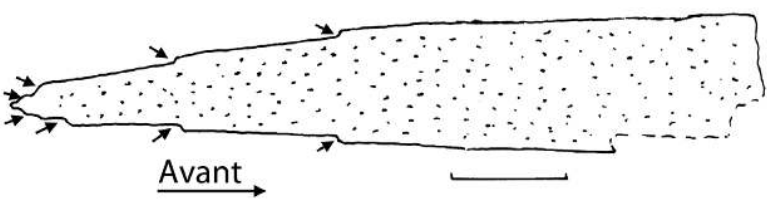

Figure 5 : Telson de crevettes grises Crangon crangon du site de Rezé (dessin Y. Gruet). L'échelle est de $1 \mathrm{~mm}$. Les flèches indiquent l'emplacement de soies qui sont tombées.

Figure 5: Telson of the sand shrimp Crangon crangon of the Rezé site (drawing Y. Gruet). Scale is $1 \mathrm{~mm}$. The arrows indicates the location where setae have fallen down.

\section{DisCUSSION - CONCLUSION}

Deux espèces de crevettes sont présentes à Ratiatum dans des proportions très dissemblables avec $96 \%$ de Palaemon longirostris pour $4 \%$ de Crangon crangon. L'espèce P. longirostris est strictement inféodée aux estuaires (Aurousseau, 1984; Béguer et al., 2010; Béguer, 2009). Ces crustacés ont pu être pêchés entre Paimbœuf et Mindin ou dans l'estuaire externe (figure 9), à partir de l'estran ou d'un petit bateau, avec un filet à maille fine ou une nasse appâtée par exemple. La faible proportion des crevettes grises $C$. crangon peut signifier que la pêche était réalisée en milieu rocheux, ou bien qu'elle se pratiquait à l'amont de l'estuaire lorsque la densité de cette espèce diminue. G. Ferronnière en 1901 atteste la présence de la crevette $P$. longirostris (sous le nom de $P$. edwardsii) jusqu'à Couéron, donc très près de Rezé
Figure 6 : Relation biométrique entre la longueur totale en millimètres et la distance entre les dents 1 et 4 du dessous du rostre de la crevette blanche Palaemon longirostris $(\mathrm{n}=54)$.

Figure 6: Biometrical relation between total length in millimetres and the distance from tooth 1 to tooth 4 under rostrum of Palaemon longirostris $(n=54)$.

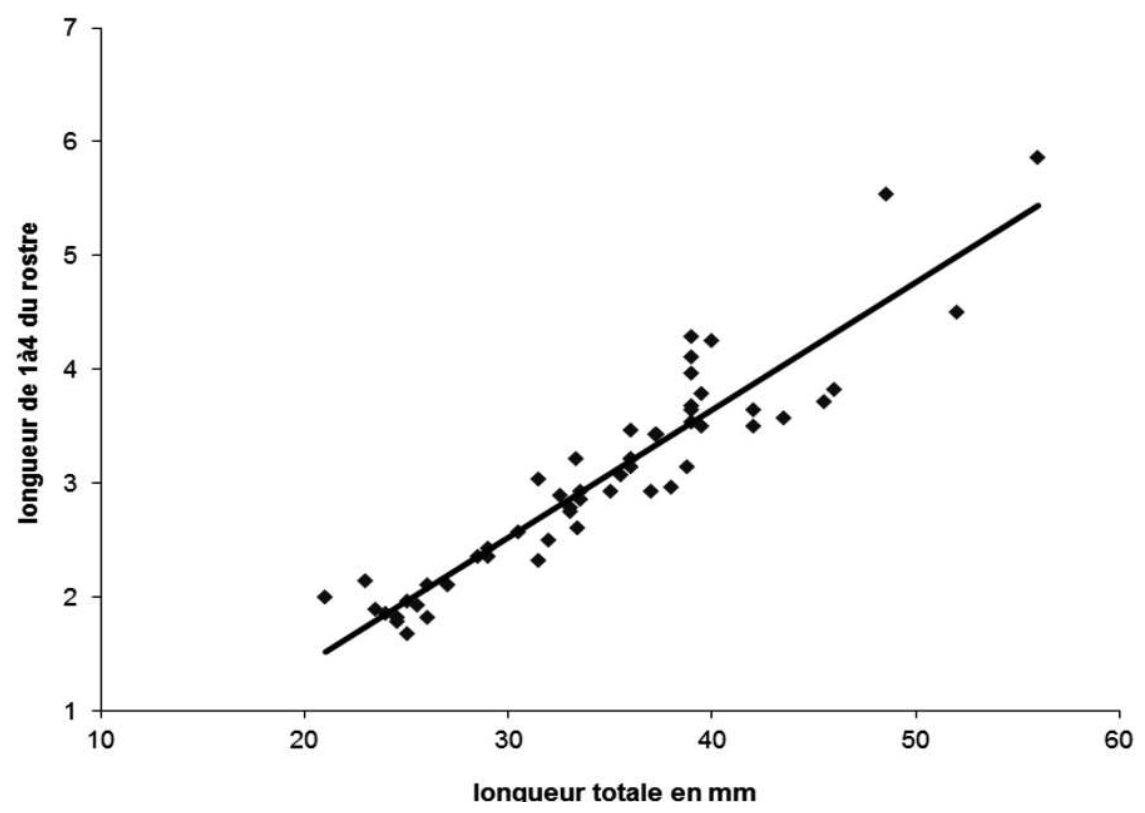

ArcheoSciences, revue d'archéométrie, 42(1), 2018, p. 7-15 


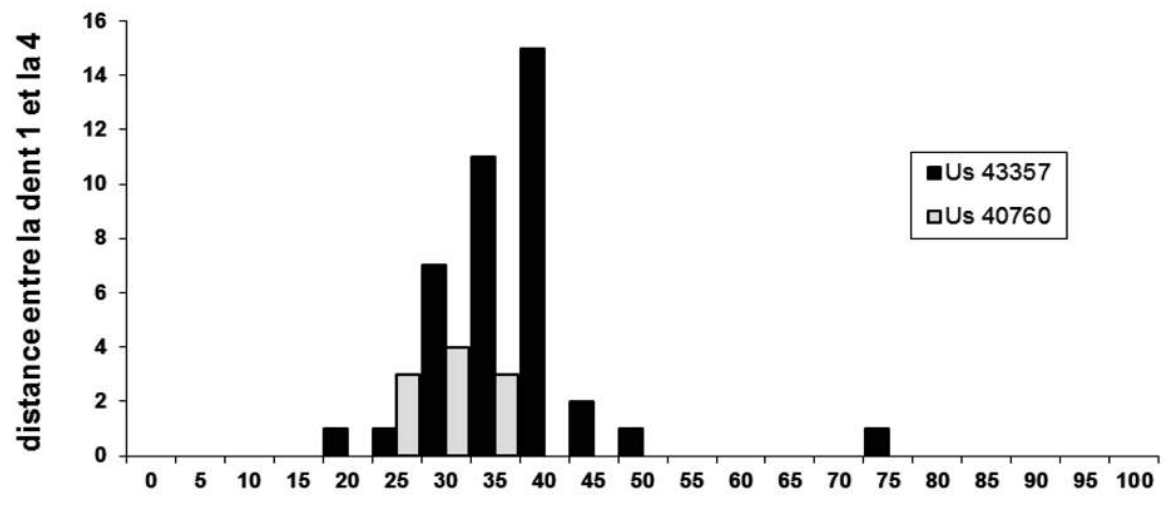

longueur totale en $\mathbf{~ m m}$
Figure 7 : Répartition de la longueur totale calculée des $P$. longirostris de Rezé pour les Us $43357(\mathrm{n}=38)$ et 40760 ( $\mathrm{n}=10)$.

Figure 7: Distribution of the total length calculated of $\mathrm{P}$. longirostris from the Rezé site for the Us $43357(n=38)$ and the Us $40760(n=10)$.
Figure 8 : Relation biométrique entre la longueur totale en $\mathrm{mm}$ et la longueur du telson en millimètres de la crevette grise Crangon crangon $(\mathrm{n}=21)$.

Figure 8: Biometrical relation between total length in millimetres and the telson lengths in $m m$ of Crangon crangon, the sandy $\operatorname{shrimp}(n=21$.

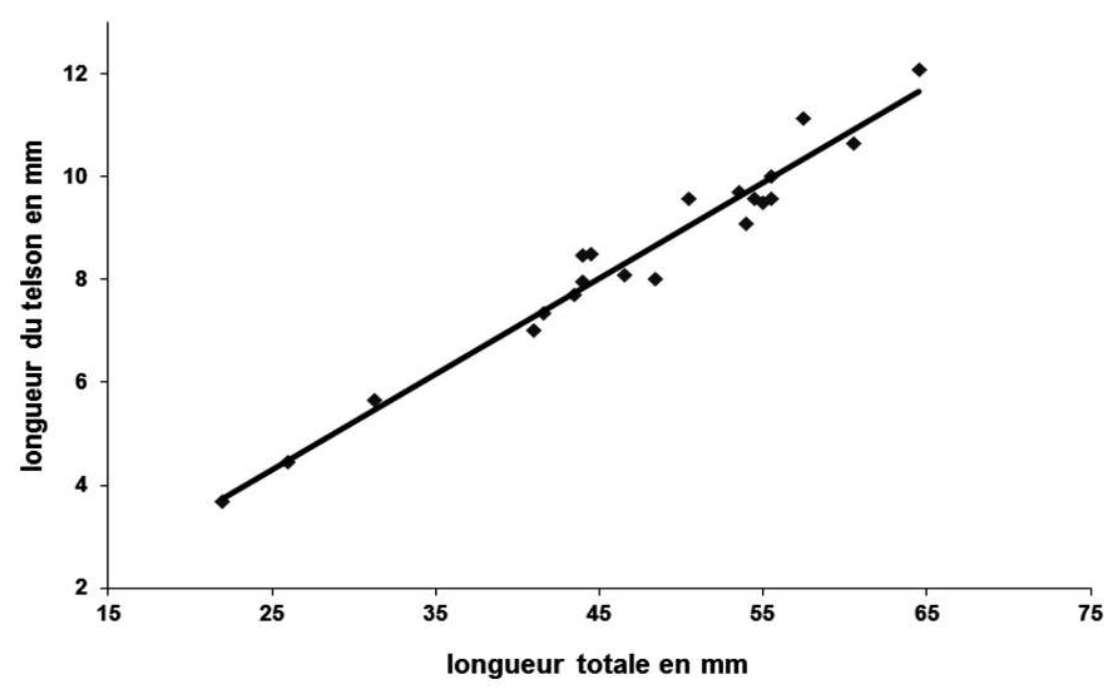

aussi dans le sens de jeunes individus probablement pêchés au printemps ou en début d'été.

Ces restes de crevettes sont systématiquement retrouvés associés à des ossements de petits poissons, étudiés en détail dans le cas de l'Us 40760 (Borvon, 2017). Ces vestiges ichthyologiques correspondent à des individus de moins de $10 \mathrm{~cm}$ pour la plupart, avec des espèces telles l'anchois, mais aussi des gobies et des syngnathes, taxons plus inhabituels en contexte archéologique. Ces restes correspondent probablement à des préparations culinaires de type sauce de poissons (garum). Il est tout à fait probable que les crevettes aient pu entrer dans la composition de ces sauces à base de poissons (Van Neer et al., 2005, 2015; Mazzocchin et Wilkens, 2013). Tous ces restes, de poissons comme de crevettes, pourraient correspondre à des résidus de filtration (Van Neer et al., 2010), de manière à expliquer leur présence dans l'environnement portuaire de Ratiatum. 
Figure 9: Carte de distribution actuelle de quelques crevettes communes vivant à proximité de la côte du Golfe de Gascogne.

Figure 9: Current distribution map of some common shrimps living near the coast of the Gulf of Biscaye.
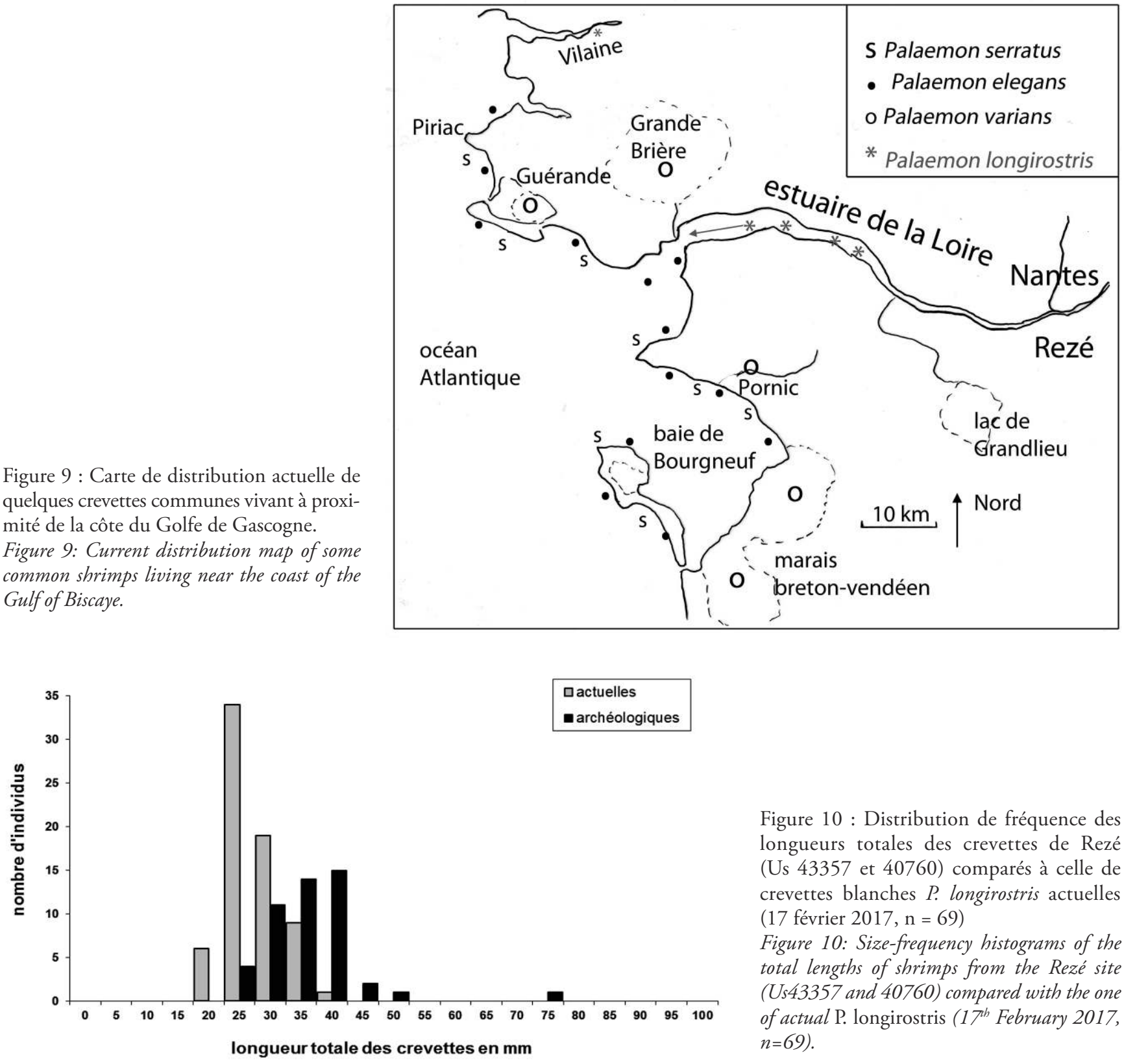

Figure 10 : Distribution de fréquence des longueurs totales des crevettes de Rezé (Us 43357 et 40760) comparés à celle de crevettes blanches $P$. longirostris actuelles (17 février 2017, $\mathrm{n}=69$ )

Figure 10: Size-frequency histograms of the total lengths of shrimps from the Rezé site (Us43357 and 40760) compared with the one of actual P. longirostris (1 $7^{\text {th }}$ February 2017, $n=69$ ).

De manière générale, la reconnaissance et l'identification des restes de crevettes sur les sites archéologiques sont rares, ce qui s'explique en partie par la fragilité de la carapace de ces animaux. À notre connaissance, seules trois mentions avec identification des espèces sont disponibles dans la littérature pour la période romaine. Il s'agit de crevettes marines Palaemon adspersus et d'une crevette d'eau douce Caridina trouvées dans des amphores romaines. La troisième étude identifie le genre Palaemon (tableau 2).

La découverte de restes de carapace de crevettes dans les niveaux portuaires du site de Rezé Saint-Lupien est assez exceptionnelle. Leur présence est rendue possible grâce aux très bonnes conditions de conservation en milieu humide, ainsi qu'à la pratique du tamisage permettant de les récolter. À l'avenir, il conviendrait de rechercher ce type de vestiges dans d'autres contextes archéologiques propices à leur conservation (milieu humide, latrines, etc.) de manière à mieux renseigner la présence jusqu'ici très discrète de ces petits crustacés. 


\begin{tabular}{|c|c|c|c|c|c|c|}
\hline & espèces & $\begin{array}{c}\text { nombre } \\
\text { d'individus }\end{array}$ & contenant & période & localisation & milieu de vie \\
\hline cette étude & $\begin{array}{c}\text { Palaemon longirostris } \\
\text { Crangon crangon }\end{array}$ & $\begin{array}{c}85 \\
\text { sédiments } \\
\text { au pied des } \\
\text { aménagements } \\
\text { portuaires }\end{array}$ & $\begin{array}{c}\mathrm{II}^{\mathrm{e}} \text {-III } \\
\text { apr. J.ècle }\end{array}$ & $\begin{array}{c}\text { Ouest de la } \\
\text { France }\end{array}$ & $\begin{array}{c}\text { eaux } \\
\text { estuariennes }\end{array}$ \\
\hline $\begin{array}{c}\text { Mazzzocchin et } \\
\text { Wilkens, 2013 }\end{array}$ & Palaemon cf. adspersus & 9 & amphore & $\begin{array}{c}\mathrm{I}^{\mathrm{er}} \text { - } \mathrm{II}^{\mathrm{e}} \text { siècles apr. } \\
\text { J.-C. }\end{array}$ & Nord de l'Italie & eaux marines \\
\hline $\begin{array}{c}\text { Van Neer } \text { et al., } \\
2015\end{array}$ & Caridina africana ou C. nilotica & 1 & amphore & $\begin{array}{c}\text { IV }{ }^{\mathrm{e}}-\mathrm{V}^{\mathrm{e}} \text { siècles } \\
\text { apr. J.-C. }\end{array}$ & Égypte & eaux douces \\
\hline Lepiksaar, 1986 & Leander sp. (= Palaemon sp.) & 13 & amphore & Antiquité & Autriche & - \\
\hline
\end{tabular}

Tableau 2 : Sites archéologiques ayant livré des restes de crevettes pour la période romaine.

Table 2: Archaeological sites that have provided shrimps remains for the Roman period.

\section{Remerciements}

Nos remerciements s'adressent aux archéologues responsables des fouilles: Martial Monteil, Jimmy Mouchard, Yves Henigfeld, Université de Nantes; Ophélie de Peretti, ville de Rezé; Rémy Arthuis, David Guitton, INRAP; ainsi qu'à Maroussia Delemarre (cabinet d'études Bio-littoral) qui nous a fourni des crevettes.

\section{Bibliographie}

Aurousseau J.-M., 1984. Eléments d'écologie de la crevette blanche, Palaemon longirostris (H. Milne Edwards, 1837), dans l'estuaire de la Gironde : dynamique de la population et production. Thèse de $3^{\text {e }}$ cycle, Université Paris VI.

Béguer M., 2009. Réponse d'une population aux forçages environnementaux et anthropiques: le suivi à long terme de la crevette Palaemon longirostris de l'estuaire de la Gironde (1979-2007). Thèse de Doctorat, Université de Bordeaux I.

Béguer M., Bergé J., Girardin M., Bö̈t Ph., 2010. Reproductive biology of Palaemon longirostris (Decapoda: Palaemonidae) from Gironde estuary (France), with a comparison with other european populations. Journal of Crustacean Biology, 30 (2), p. 175-185.

Borvon A., 2016. Analyse des vestiges osseux de Rezé SaintLupien, zone 4, campagne de fouilles 2015. In D. Guitton, J. Mouchard (coord.), L'agglomération antique de Rezé (LoireAtlantique). Le quartier Saint-Lupien. Rapport de fouille programmée 2015. Les aménagements de berge (zone 4). Tome 2 - Études spécialisées, SRA Pays de la Loire, Nantes, p. 59-100.

Borvon A., 2017. Les poissons du port romain de Ratiatum (Rezé, Loire-Atlantique) : espèces consommées, sauces et techniques de pêche. In R. Gonzalez Villaescusa, K. Schörle, F. Gayet, F. Rechin (coord.), L'exploitation des ressources maritimes de
l'Antiquité. Activités productives et organisation des territoires. XXXVII Rencontres Internationales d'Archéologie et d'Histoire d'Antibes et XII Colloque de l'Association AGER, octobre 2016, APDCA, Antibes, p. 23-40.

Boutelier P., Gouleau D., Nikodic J., Ottmann F., 1982. Les échanges transversaux entre le chenal et les vasières latérales dans l'estuaire de la Loire. Mémoire de la Société Géologique de France, 144, p. 101-110.

Bouvier E.L., 1940. Décapodes Marcheurs. Paris, Lechevalier et Fils, coll. "Faune de France; 37 ».

Ferronnière G., 1901. Études biologiques sur les zones supralittorales de la Loire-Inférieure. Bulletin de la Société des Sciences Naturelles de l'Ouest de la France, 1, p. 1-453.

Gruet Y., Baudet Y., 2007. Marais de Brière et du Brivet : des eaux saumâtres sous influence de l'estuaire de la Loire. Aestuaria, 10 (Pour une gestion durable des zones humides : l'exemple des parcs naturels régionaux), p. 95-122.

Guitton D., Mouchard J. (coord.), 2015. L'agglomération antique de Rezé (Loire-Atlantique). Le quartier SaintLupien. Rapport de fouille programmée 2014. Les aménagements de berge (zone 4). SRA Pays de la Loire, Nantes.

Guitton D., Mouchard J. (coord.), 2016. L'agglomération antique de Rezé (Loire-Atlantique). Le quartier SaintLupien. Rapport de fouille programmée 2015. Les aménagements de berge (zone 4). SRA Pays de la Loire, Nantes.

LepiksaAR J. 1986. Tierreste in einer römischen Amphore aus Salzburg (Mozartplatz 4). Bayerische Vorgeschichtsblätter, 51, p. $163-185$.

Marchand J., 1981. Observations sur l'écologie de Crangon crangon (Linné) et Palaemon longirostris H. Milne Edwards (Crustacea, Decapode, Natantia) dans l'estuaire interne de la Loire (France). Vie et Milieu, 31 (1), p. 83-92.

Marchand J., Alliot A., 1981. Observations sur l'écologie des populations de trois espèces de Crustacés Décapodes nageurs 
en Loire Atlantique : Palaemonetes varians (Leach), Palaemon longirostris H. Milne Edwards et Crangon crangon (Linné). Bulletin de la Société de Sciences naturelles de l'Ouest de la France, 3 (4), p. 184-201.

Mazzocchin S., Wilkens B., 2013. Fish and Crustaceans from a Roman Amphora in Northern Italy. Archaeofauna, 22, p. 105111.

NoËL P.-Y., 1992. Clé préliminaire d'identification des Crustacea Decapoda de France et des principales autres espèces d'Europe. Paris, Muséum national d'Histoire naturelle, coll. « Patrimoines naturels; 9 ".

SChwartz D., 1963. Méthodes statistiques à l'usage des médecins et des biologistes. Paris, Flammarion.

Smaldon G., 1979. British Coastal Shrimps and Prawns. Synopses of the British Fauna (New series). London, The Linnean Society of London/Academic Press.

UDEKEM D'ACOz C. (d'), 1999. Inventaire et distribution des crustacés décapodes de l'Atlantique nord-oriental, de la Méditerranée et des eaux continentales adjacentes au nord de $25^{\circ} \mathrm{N}$. Paris, Muséum national d'Histoire naturelle/Société Nationale de Protection de la Nature.

Van Neer W., Ervynck A., Monsieur P., 2010. Fish bones and amphorae: evidence for the production and consumption of salted fish products outside the Mediterranean region. Journal of Roman Archaeology, 23, p. 161-195.

Van Neer W., Wouters W., Ervynck A., Maes J., 2005. New evidence from a Roman context in Belgium for fish sauce locally produced in northern Gaul. Archaeofauna, 14, p. 171182.

Van Neer W., Wouters W., Codina Reina D., Fournet J.-L., Preiss S., 2015. Découverte de deux salaisons de poissons à Oxyrhyncus, el-Bahnasa, Égypte. Nova Studia Aegyptiaca, IX, p. 567-578.

Zariquiey Alvarez R., 1968. Crustàceos decâpodos ibéricos. Barcelona, Investigacion Pesquera. 